\title{
Modelos basados en variables meteorológicas para estimar el progreso epidémico de la bacteriosis del nogal en variedades de distinto comportamiento
}

\author{
Silvana Andrea Seta ${ }^{1} \mathbb{\oplus}$, Ricardo Moschini ${ }^{2} \mathbb{0}$, Mirian del Pilar Gonzalez ${ }^{1.3}$. $^{\circ}$
}

${ }^{1}$ Facultad de Ciencias Agrarias. Universidad Nacional de Rosario, Campo Experimental Villarino, C.C. 14 (S2125ZAA) Zavalla, Santa Fe, Argentina. ${ }^{2}$ Instituto de Clima y Agua. CIRN INTA Castelar. Los Reseros y Las Cabañas s/n. CP:1686. Hurlingham Bs. As. Argentina. ${ }^{3}$ Carrera Investigador Científico Consejo Investigaciones UNR, Universidad Nacional de Rosario, Campo Experimental Villarino, C.C. 14 (S2125ZAA) Zavalla, Santa Fe, Argentina.

Autor para correspondência: Seta Silvana Andrea (sil.seta@gmail.com)

Data de chegada: 23/09/2019. Aceito para publicação em: 01/06/2020.

$10.1590 / 0100-5405 / 234402$

\section{RESUMEN}

Seta, S.A.; Moschini, R.; Gonzalez, M.del P.. Modelos basados en variables meteorológicas para estimar el progreso epidémico de la bacteriosis del nogal en variedades de distinto comportamiento. Summa Phytopathologica, v.47, n.1, p.16-20, 2021.

La bacteriosis del nogal es causada por Xanthomonas arboricola $p v$. juglandis. En Zavalla (Santa Fe), la actividad nogalera se centra en la producción de plantas injertadas en vivero. El presente trabajo tuvo por objetivo cuantificar el efecto ambiental sobre el desarrollo epidémico de la bacteriosis del nogal en variedades susceptibles y de mejor comportamiento a la enfermedad y elaborar modelos matemáticos que permitan predecir la severidad de la misma. En la localidad de Zavalla se realizaron observaciones de severidad (Sev\%) de la bacteriosis cada siete días en cuatro variedades de nogal (tres susceptibles (sus=1): Chandler, Davis y Tulare y una de mejor comportamiento: Franquette $($ sus $=0)$ ), desde el fin de la primavera hasta los últimos días de febrero $(\mathrm{N}=76)$ durante tres campañas (2010/11, 2011/12 y 2012/13). Las variables regresoras meteorológicas se calcularon en los respectivos lapsos de 15 días previos a cada observación de tasa de incremento diario epidémico $\left(\mathrm{Tid} \%=\operatorname{Sev} \%-\operatorname{Sev} \%_{\mathrm{t}-1} / 7\right)$. Las variables mejor correlacionadas $\left(\mathrm{r}_{\mathrm{k}}\right.$ : coeficiente de correlación de Kendall
Tau-b) con los niveles de bacteriosis (severo:Tid $\%>0,1367$; moderado a nulo: Tid $\%<=0,1367$, siendo 0,1367 el percentil $40 \%$ de los datos observados), para los dos tipos de variedades (sus $=1$ y sus $=0$ ), fueron DPr $>9$ (días con precipitación $>9 \mathrm{~mm} ; \mathrm{r}_{\mathrm{k}}=0,434$ ) y DMojro (días sin lluvia, con mojado atribuible al rocío por ocurrencia de humedad relativa del aire mayor a $82 \% ; r_{k}=0,326$ ). El modelo logístico integrado por DPr $>9$ y DMojro clasificó correctamente 62 casos de los 76 analizados en las tres campañas (precisión de predicción: PPred $=81,5 \%$ ), permitiendo cuantificar el efecto ambiental en ambos tipos de variedades de nogal analizadas. El modelo integrado por DPr $>9$ y DMojt (suma los días de mojado por rocío (DMojro) con los originados por precipitación (DMojpr)) logró una precisión de predicción de $80,3 \%$. Ambas variables meteorológicas estarían expresando el requerimiento de agua libre de la bacteria para la infección (DMojt) y de energía en las lluvias (DPr>9) para favorecer la dispersión bacteriana.

Palabras adicionales: Xanthomonas arboricola pv juglandis, epidemiología, factores climáticos, Juglans regia, Argentina.

\section{ABSTRACT}

Seta, S.A.; Moschini, R.; Gonzalez, M. del P. Models based on meteorological variables to estimate the epidemic progress of the walnut bacteriosis in varieties of different behavior. Summa Phytopathologica, v.47, n.1, p.16-20, 2021.

Walnut Blight is caused by Xanthomonas arboricola pv. juglandis. In Zavalla (Santa Fe), walnut production is focused on the grafted plants in nurseries. The objective of this work was to quantify the environmental effect on the epidemic development of walnut blight, in susceptible varieties and in varieties with better behaviour to the disease, and to develop mathematical models to predict the severity of the disease In the locality of Zavalla observations of severity (Sev\%) of walnut blight were made every seven days in four walnut varieties (three susceptible (sus=1): Chandler, Davis and Tulare and one with better performance: Franquette (sus $=0$ )), from the end of spring until the last days of February $(N=76)$, during three crop seasons $(2010 / 11,2011 / 12$ and 2012/13). Weather regression variables were calculated in the respective 15-day periods prior to each observation of daily epidemic increase rate (Tid $\%=\operatorname{Sev} \% \mathrm{t}-\mathrm{Sev} \% \mathrm{t}-1 / 7)$. The variables best correlated (rk: Kendall Tau-b correlation coefficient) with bacterial levels (severe: Tid $\%>0.1367$; moderate to nil) were $\operatorname{Tid} \%<=0.1367$, with 0.1367 being the $40 \%$ percentile of the observed data), for both types of varieties (sus=1 and sus $=0$ ), were DPr $>9$ (days with precipitation $>9 \mathrm{~mm} ; \mathrm{rk}=0.434$ ) and DMojro (days without rain, with wetness attributable to dew due to the occurrence of relative air humidity greater than $82 \%$; $\mathrm{rk}=0.326$ ). The logistic model integrated by $\operatorname{DPr}>9$ and DMojro correctly classified 62 cases of the 76 analyzed in the three crop seasons (prediction accuracy: PPred $=81.5 \%$ ), allowing to quantify the environmental effect in both types of walnut varieties analyzed. The model integrated by DPr $>9$ and DMojt (summing the days of wetting by dew (DMojro) with those originated by precipitation (DMojpr)) achieved a prediction accuracy of $80.3 \%$. Both meteorological variables would be expressing the bacterial free water requirement for infection (DMojt) and energy requirement in rainfall $(\mathrm{DPr}>9)$ to provide bacterial dispersion.

Keywords: Xanthomonas arboricola pv juglandis, epidemic development, environmental effect, Juglans regia, Argentina.

\section{RESUMO}

Seta, S.A.; Moschini, R.; Gonzalez, M.del P. Modelos baseados em variáveis meteorológicas para estimar o progresso epidêmico do bacteriose de noz em variedades de comportamento diferente. Summa Phytopathologica, v.47, n.1, p.16-20, 2021.

A bacteriose da nogueira é causada por Xanthomonas arboricola pv. juglandis. Em Zavalla (Santa Fe), o cultivo da nogueira concentra-se na produção de plantas enxertadas em viveiro. O objetivo deste trabalho foi quantificar o efeito do ambiente no desenvolvimento epidêmico da bacteriose da nogueira em variedades susceptíveis e de melhor comportamento e elaborar modelos matemáticos que permitam prever a sua severidade. En Zavalla foram feitas observações da severidade da bacteriose $(\mathrm{Sev} \%)$ a cada sete dias em quatro variedades de nogueiras 
(três susceptíveis (sus=1): Chandler, Davis e Tulare e uma de melhor comportamento: Franquette (sus=0)), desde o final da primavera até os últimos dias de fevereiro $(\mathrm{N}=76)$, durante três safras $(2010 / 11,2011 / 12$ e 2012/13), As variáveis de regressão meteorológica foram calculadas nos respectivos intervalos de 15 dias antes de cada observação da taxa diária de progresso da epidemia (Tid\%=Sev\%t-Sev\%t-1/7). As melhores variáveis correlacionadas (rk: coeficiente de correlação de Kendall Tau-b) com a severidade da bacteriose (severo:Tid $\%>0,1367$; moderado a nulo: Tid $\%<=0,1367$, sendo 0,1367 o percentil de $40 \%$ dos dados observados), para os dois tipos de variedades (sus=1 e sus=0), foram $D P r>9$ (dias com precipitação $>9 \mathrm{~mm}$; rk=0,434) e DMojro (dias sem chuva, com molhamento devido ao orvalho pela ocorrência de umidade relativa do ar maior que $82 \%$; $\mathrm{rk}=0,326)$. O modelo logístico integrado por $\mathrm{DPr}>9$ e DMojro classificou corretamente 62 casos dos 76 analisados nas três safras (precisão de previsão: PPred=81,5\%), permitindo quantificar o efeito do ambiente em ambos os tipos de variedades. O modelo integrado por DPr $>9$ e DMojt (soma dos dias com molhamento foliar pelo orvalho (DMojro) com os originados por precipitação pluvial (DMojpr)) atingiu uma precisão de previsão de $80,3 \%$. Ambas as variáveis meteorológicas estariam expressando a necessidade de água livre para a infecção (DMojt) e o efeito mecânico da precipitação pluvial ( $\mathrm{DPr}>9$ ) na dispersão bacteriana. Com o uso do melhor modelo podem ser reduzidas as pulverizações com bactericida.

Palavras-chave: (5) Xanthomonas arboricola pv juglandis, epidemiologia, fatores climáticos, Juglans regia, Argentina.

El cultivo del nogal en la Argentina, fue introducido al país por las colonizaciones españolas, y se extendió principalmente en las provincias andinas y en los valles intermontanos de altura donde las plantas se desarrollaron con las mejores condiciones ambientales y a la vez colaboraron en el crecimiento de varias economías regionales. Las áreas tradicionales de producción se localizan en las provincias de Mendoza, Catamarca, La Rioja, Río Negro y Neuquén y por último se agregó la provincia de San Juan como productora.

A mediados de la década del 90 y hasta el año 2000, el sector emprendió un marcado proceso de reconversión orientado a reemplazar las variedades criollas (originadas de semillas, en las que no se consideraban aspectos vinculados con la rentabilidad, pronto inicio de cosecha o calidad del fruto, con frutos de tamaño pequeño o mediano y una coloración de pulpa ámbar a ámbar oscuro, baja relación pulpacáscara) por las denominadas finas (ranquette, Howard y Chandler, y otras variedades californianas como Sunland, Serr, Chico, Davis)

El proceso de reconversión consistió básicamente en el replantado en Mendoza, el reinjertado escalonado en el tiempo en las provincias de Catamarca y La Rioja y el aumento de la densidad de los montes mediante el intercalado de plantas, pasándose de 50 a 200 plantas/ha, con rápida entrada en producción. Los primeros montes reconvertidos ya comenzaron a producir, con rendimientos de $3.500 \mathrm{Kg}$./ha. Argentina, junto a Chile, es uno de los principales países productores de nueces del Hemisferio Sur. Actualmente la producción nacional abastece parcialmente la demanda del mercado interno, aunque una gran proporción carece de estandarización y presenta calidad variable (15).

Siendo el sur de la provincia de Santa Fe una zona con suelos de tipo argiudol vértico y con alta humedad, una de las enfermedades más importantes que se producen en zonas húmedas es el tizón bacteriano (16). El tizón bacteriano o bacteriosis del nogal, o peste negra es causada por la bacteria Xanthomonas arboricola pv. juglandis (Xaj). Las variedades californianas de nogal son más susceptibles a la bacteriosis porque son más precoces que las tradicionales como Franquette.

La bacteria ingresa a la planta a través de los estomas, lenticelas en frutos, estigmas y heridas. Prácticamente todos los órganos aéreos de la planta pueden ser afectados, pero los daños más importantes son los que se producen en los frutos. Sobrevive en forma primaria en yemas dormidas. La infección de las yemas es variable y va desde cantidades indetectables hasta $1 \times 10^{7}$ células por yema (11). Las flores son usualmente infectadas apenas aparecen en la primavera, aun después de que los estigmas son receptivos al polen. Muchas flores y frutos pequeños afectados caen al suelo. Bajo condiciones favorables $X$. arboricola pv juglandis se multiplica en yemas, en hojas y frutos jóvenes. Este mecanismo de dispersión no es claro, pero probablemente la bacteria es transportada por la lluvia, por insectos, además del polen. El polen, llevado por el viento a largas distancias, traslada la bacteria desde flores estaminadas infectadas (12).

Los síntomas característicos son lesiones superficiales en fruta inmadura que comienzan como manchas pequeñas y acuosas las cuales con posterioridad se oscurecen y agrandan rápidamente. La infección finalmente resulta en lesiones oscuras de alrededor de 2,5 cm. El daño causado principal se debe a la reducción de la productividad por la caída precoz de frutos o por que la fruta infectada resulta no comerciable.

La dinámica del desarrollo bacteriano es poco cíclica y muy errática. La humedad es fundamental para el desarrollo de la bacteria. La infección es favorecida por agua libre, lluvias, humedad relativa alta y temperaturas templadas, superiores a $\operatorname{los} 15^{\circ} \mathrm{C}$ en la primavera.

El control de la enfermedad es de carácter preventivo y consiste en la aplicación semanal de bactericidas derivados del cobre o antibióticos derivados de la kasugamicina $(5,14,17)$. Todos los cultivares comerciales son susceptibles, pero aquellos de brotación tardía como Hartley y Franquette pueden evitar la enfermedad en climas mediterráneos, en los cuales las lluvias terminan abruptamente a fines de primavera. Adaskaveg et al. $(1,2,3)$ y Arquero et al. (4) evaluaron el efecto de distintos factores en la incidencia de la enfermedad, encontrando diferencias en relación a la susceptibilidad para las distintas variedades, y a las distintas zonas del canopeo en las diferentes plantas. La disminución del número de aplicaciones de bactericidas tendría efectos favorables en la reducción de costos del cultivo y beneficiaría la sustentabilidad económica y ambiental del sistema. En el año 2000 se inició un modelo de pronóstico de la bacteriosis del nogal denominado XanthoCast ${ }^{\mathrm{TM}}(1,2)$ en la Universidad de California. Este sistema está desarrollado con todos los componentes del triángulo de la enfermedad (hospedante, patógeno y ambiente). El componente hospedante está definido por el estado fenológico y por la resistencia diferencial entre los cultivares. En el componente patógeno, el inóculo aparece como el parámetro más predecible para estimar ya que es endémico en plantaciones de nogales a lo largo de toda California. La incidencia de la enfermedad del año anterior es un excelente indicador del inóculo potencial de un monte para la siguiente estación, porque la bacteria tiene una alta capacidad reproductiva bajo condiciones ambientales favorables como agua libre y temperaturas primaverales superiores a $15^{\circ} \mathrm{C}$. En estudios comparativos de campo el modelo Xantho Cast permitió la reducción del número de aplicaciones de bactericidas en montes en producción. El conocimiento de la epidemiología de esta enfermedad, el desarrollo de la enfermedad en el tiempo, su relación con las condiciones ambientales y la variabilidad de las cepas existentes permitirían el establecimiento de pautas generales para el manejo efectivo de la enfermedad.

El presente trabajo tuvo por objetivo cuantificar el efecto ambiental sobre el desarrollo epidémico de la bacteriosis del nogal (Zavalla; Santa Fe), en variedades susceptibles y de mejor comportamiento a la 
enfermedad y elaborar modelos matemáticos que permitan predecir la severidad de la misma.

\section{MATERIALES Y MÉTODOS}

Durante tres estaciones de crecimiento (2010/11, 2011/12, 2012/13), fueron realizadas observaciones semanales de severidad (Sev\%: área foliar afectada/área foliar evaluada x 100) de la bacteriosis, en 8 plantas de nogal por parcela de las variedades susceptibles Chandler, Davis y Tulare (sus=1) y de mejor comportamiento (Franquette: sus=0) del monte frutal del Campo Experimental de la Facultad de Cs. Agrarias de la UNR ( Zavalla, Santa Fe, Long. O $60^{\circ} 53^{`}$; Lat. S $33^{\circ} 01^{`}$ y altitud de $50 \mathrm{~m}$ sobre el nivel del mar). En cada fecha se tomó el valor mediana de las observaciones realizadas en el total de plantas. Las observaciones semanales de severidad se realizaron desde el fin de primavera a los últimos días de febrero. A lo largo de cada curva de progreso epidémico se calcularon las tasas de incremento diaria $\left(\mathrm{Tid} \%=\mathrm{Sev} \%_{\mathrm{t}}-\mathrm{Sev} \%_{\mathrm{t}-1} /\right.$ 7) por fecha de monitoreo y para los tres ciclos de cultivo. El total de valores de Tid\% $(\mathrm{N}=76)$ fue utilizado para el desarrollo de modelos logísticos predictivos. En el caso de las variedades susceptibles se calculó la media de las tres observaciones de Tid\% correspondientes a cada variedad.

\section{Desarrollo de los modelos logísticos:}

Variable dependiente: probabilidad de ocurrencia de niveles categorizados de tasa de incremento de severidad de la bacteriosis. La tasa de incremento epidémico diario (Tid\%) surgió de la diferencia entre el valor acumulado (Sev\%) en el tiempo t y el valor en $\mathrm{t}-1$, dividido el número de días entre las dos observaciones (7 días).

Categorización: Ordenando en forma ascendente los valores de tasa de incremento epidémico diario ( $\mathrm{Tid} \%)$ disponibles $(\mathrm{N}=76)$ para las tres curvas epidémicas obtenidas por tipo de variedad, el percentil 40 $\%$ alcanzó el valor $0,1367 \%$. Este umbral fue utilizado para categorizar binariamente las observaciones de Tid\% en: severa(S): TI $>0,1367 \%$ y moderada a nula $(\mathrm{M})$ : $\mathrm{Tid}<=0,1367 \%$.

\section{Variables meteorológicas regresoras}

La estación meteorológica convencional de Zavalla registró diariamente la temperatura máxima $\left(\mathrm{Tx}:{ }^{\circ} \mathrm{C}\right)$ y mínima $\left(\mathrm{Tn}:{ }^{\circ} \mathrm{C}\right)$, precipitación pluvial (Pr:mm) y humedad relativa (HR:\%). La temperatura media diaria (Tm) fue calculada como la semisuma de las temperaturas máxima y mínima. La estación solo registra las temperaturas máxima y mínima diarias, no disponiendo de registros térmicos horarios. Basados en estos elementos se construyeron las siguientes variables meteorológicas simples: $\mathbf{D P r}>\mathbf{9}$ : total de días con precipitación $>9 \mathrm{~mm}$. Prac: total acumulado en $\mathrm{mm}$ de precipitaciones diarias; DMojro días sin lluvia $(<0,2 \mathrm{~mm})$, con mojado atribuible al rocío por ocurrencia de humedad relativa del aire mayor a $82 \%$. DMojpr: días con precipitación ( $>=0,2 \mathrm{~mm})$. DMojt: se suman los días de las variables DMojro y DMojpr. DHR: días con humedad relativa del aire mayor a $81 \%$. TxM: Tx media; TnM: Tn media; DT: total de días con $\mathrm{Tx}<24^{\circ} \mathrm{C}$ y $\mathrm{Tn}>11^{\circ} \mathrm{C}$.

\section{Variable discreta}

Variable discreta binaria que tiene en cuenta el efecto del comportamiento varietal respecto a la bacteriosis (sus=1 para las variedades susceptible $(\mathrm{N}=38)$ y sus $=0$ para la variedad Franquette $\mathrm{N}=38$ )
Lapso de cálculo de las variables: en los 15 días previos a cada observación de Tid\% a lo largo de cada curva epidémica analizada

\section{Análisis estadístico}

- Cálculo de coeficientes de correlación no paramétrica (r) de Kendall Tau-b (Proc Freq del SAS) entre los niveles epidémicos y las variables meteorológicas descriptas

- Mediante el procedimiento Logistic del SAS se ajustaron modelos de regresión logística a los datos de respuesta binaria (método de máxima verosimilitud). Si se asume que $\mathrm{PrS}$ es la probabilidad de observar un nivel de Tid\% severo (S), el modelo logístico, con $\mathrm{X}_{1}$ y $\mathrm{IX}_{1} \mathrm{X}_{2}$ (interacción) como predictores, puede ser escrito como: $\ln (\mathrm{PrS} / 1$ $\operatorname{PrS})=\beta 0+\beta 1 \mathrm{X}_{1}+\beta 2 \mathrm{IX}_{1} \mathrm{X}_{2}$, en el cual $\beta 0 \mathrm{a} \beta 2$ son parámetros $\mathrm{y} \ln$ es el logaritmo natural. Resolviendo la expresión $\operatorname{Exp}(\ln (\operatorname{PrS} / 1-\operatorname{PrS}) /$ $(1+\operatorname{Exp}(\ln (\operatorname{PrS} / 1-\operatorname{PrS}))$ se obtiene el valor de PrS. PrL $=1-\operatorname{PrS}$ (respuesta binaria). El método de selección de variables Stepwise del Proc Logistic fue usado para obtener el modelo más apropiado, haciendo jugar las variables meteorológica y discreta, fijando niveles de significancia de 0,25 para entrar (NSE) y permanecer (NSP) en el modelo. Mediante el procedimiento Freq del SAS se confeccionaron tablas de contingencia para calcular el número de casos en los cuales coincide el nivel de Tid\% observado ( $\mathrm{S}$ o M) con el predicho. La precisión de predicción (Prec\%) representa el porcentaje de casos analizados $(\mathrm{N}=76)$ correctamente clasificados con nivel de Tid\% S o M.

Tanto el coeficiente de correlación de Kendal Tau-b como la precisión de predicción de los modelos logísticos desarrollados fueron tomados en cuenta para la selección final de la longitud del período en el cual se procesan las variables meteorológicas, de los umbrales de dichas variables y del valor percentil utilizado en la categorización de la variable respuesta.

\section{RESULTADOS Y DISCUSIÓN}

En la Tabla 1 se presentan los coeficientes de correlación (r) de Kendall Tau-b obtenidos entre las variables meteorológicas analizadas y los niveles epidémicos de la bacteriosis. El valor máximo de $r_{k}(0,4342)$ correspondió a la variable $\mathrm{DPr}>9$ que cuenta el número de días totales con precipitación $>9 \mathrm{~mm}$.

Tabla 1. Coeficientes de correlación (r) de Kendall Tau-b de variables meteorológicas en relación a los niveles epidémicos (severo y moderado a nulo).

\begin{tabular}{cccc}
\hline Variable & rk & Variable & rk \\
\hline DPr $>9$ & 0,434 & Prac & 0,277 \\
DMojro & 0,326 & DMojpr & 0,296 \\
DMojt & 0,344 & DHR & 0,336 \\
TnM & 0,169 & TxM & 0,04 \\
\hline DT & 0,159 & &
\end{tabular}

DPr>9: total de días con precipitación $>9 \mathrm{~mm}$. Prac: total acumulado en $\mathrm{mm}$ de precipitaciones diarias; DMojro días sin lluvia $(<0,2 \mathrm{~mm})$, con mojado atribuible al rocío por ocurrencia de humedad relativa del aire mayor a $82 \%$. DMojpr: días con precipitación ( $>=0,2 \mathrm{~mm})$. DMojt: se suman los días de las variables DMojro y DMojpr. DHR: días con humedad relativa del aire mayor a 81\%. TxM: Tx media; TnM: Tn media; DT: total de días con $\mathrm{Tx}<24^{\circ} \mathrm{C} \mathrm{y}$ $\mathrm{Tn}>11^{\circ} \mathrm{C}$. Las variables son calculadas en el lapso de 15 días previos a cada observación de Tid\% a lo largo de cada curva epidémica. 
Tabla 2. Modelos logísticos para estimar la probabilidad de ocurrencia de cada categoría epidémica: severa (S) y moderada a nula (M), en base a variables meteorológicas simples y una discreta que fija el comportamiento varietal (sus). Se presenta por modelo la precisión de predicción: \% de casos (76) correctamente clasificados.

\begin{tabular}{lll}
\hline Modelo & Ecuaciones del Modelo & Precisión de predicción \% \\
I & $\mathrm{Ec} 1=-2,189+1,024 * \mathrm{DPr}>9+0,609 * \mathrm{DMojro}+1,7147 *$ sus & 81,5 \\
& $\mathrm{Ec} 1=-2,3282+0,9499 * \mathrm{DPr}>9+0,1833^{*} \mathrm{DMojt}+1,6786 *$ sus & $(62 / 76 * 100)$ \\
II & & 80,0 \\
\hline
\end{tabular}

Ec1 $=\ln (\operatorname{PrS} / 1-\operatorname{PrS})$; Resolviendo la expresión Exp $(\ln (\operatorname{PrS} / 1-\operatorname{PrS}) /(1+\operatorname{Exp}(\ln (\operatorname{PrS} / 1-\operatorname{PrS}))$ se obtiene el valor de PrS (probabilidad de observar una tasa epidémica severa (S). Ln es el logaritmo natural. PrM la probabilidad de observar una epidemia moderada a nula $(\mathrm{M})=1-\operatorname{PrS}$.

Mediante el procedimiento Proc Logistic del SAS se ajustó el modelo I que incluye, además de dos variables meteorológicas, una variable discreta binaria que tiene en cuenta el efecto del comportamiento varietal respecto a la bacteriosis (sus $=1$ y sus $=0$ ) (Tabla 2). Este modelo resultó el más apropiado de acuerdo al procedimiento stepwise corrido con todas las variables meteorológicas y la discreta (sus), con un nivel de significancia para entrar al modelo de 0,25 . Cuando se desechó el efecto de la variable 'sus' que tiene en cuenta el comportamiento varietal, la precisión de predicción del modelo logístico decreció a 69,7\% (53 observaciones clasificadas correctamente). El modelo II que integra a DPr $>9$ y DMojt permitió clasificar correctamente 61 casos de los 76 observados. Este modelo incluye el efecto de lluvias con un mínimo milimetraje $(>9 \mathrm{~mm})$ para favorecer la dispersión bacteriana más los días donde se dispondría de agua libre originada en el rocío (DMojro) y por ocurrencia de precipitaciones $(>0.2 \mathrm{~mm}$ ) (DMojpr).

En forma similar al modelo de pronóstico de la bacteriosis del nogal denominado XanthoCast ${ }^{\mathrm{TM}}(1,2,3)$ de la Universidad de California, que considera los componentes del triángulo de la enfermedad (hospedante, patógeno y ambiente), el modelo desarrollado en este trabajo, considera también variables meteorológicas y hace referencia a la susceptibilidad o no del hospedante, como forma de predecir futuras epifitias. El modelo XanthoCast ${ }^{\mathrm{TM}}$ fue iniciado en el año 2000 como un modelo de pronóstico para el tizón bacteriano en cultivares Vina y Ashley de nogales, en California, EEUU. Dicho modelo incluía duración del período húmedo y temperatura, los dos parámetros microambientales críticos para el desarrollo de la enfermedad, tanto en cámaras de crecimiento como en estudios a campo. El modelo fue validado en 2004, 2005 y 2006 con su sitio web correspondiente. Con el uso de dicho modelo se redujo el número de aplicaciones con bactericidas comparándose con las aplicaciones siguiendo el calendario tradicionalmente utilizadas. Con el correr de los años dicho modelo se ha ido validando en diferentes condiciones climáticas y se ha adaptado a otros factores climáticos como precipitaciones, en lugar de follaje mojado (3).

Numerosos autores, entre ellos los ya citados, Lang y Evans (10), Giraud et al. (9) y otros, sugieren el desarrollo de modelos de predicción de epifitias del nogal para lograr que el manejo de esta especie sea más sustentable y cuidadoso del ambiente.

En 2002, Costa et al. (8), fundamentaron el uso de modelos epidemiológicos, como herramienta eficiente para el estudio de enfermedades a nivel de poblaciones y comunidades. En cuanto a prevención de epifitias éstos cuantifican el probable inicio o desarrollo futuro de enfermedades a partir de información del clima, del hospedante y del patógeno. Según estos autores los modelos cumplen con algunos objetivos básicos como la reducción del número de aplicaciones de productos químicos, un manejo más eficiente de la enfermedad, disminución del riesgo de ocurrencia de epidemias severas y reducción de la polución ambiental, por el menor uso de químicos.

Por su parte Moschini et al. (13), en 2010 trabajaron en la identificación de variables meteorológicas durante la primavera en la localidad de Bella Vista (Corrientes, Argentina) para predecir niveles ordinales de intensidad de la Cancrosis de los cítricos en media estación. El mejor modelo logístico ajustado incluyó además de variables meteorológicas una variable discreta binaria que tuvo en cuenta la distancia a una cortina rompeviento. en un modelo en relación a la intensidad de la Cancrosis de los cítricos. Para este mismo modelo, aplicado luego en la región citrícola de España, también para Cancrosis, se debieron agregar otras variables para explicar la intensidad de la enfermedad.

Bombelli et al (6) y Bombelli et al (7), desarrollaron modelos logísticos para predecir la severidad de manchas foliares causadas por Alternaria tenuissima (Kunze:Fr) en plantaciones de arándano en tres localidades argentinas: San Pedro, Concordia y Gualeguaychu. Uno de los modelos integró la interacción de días con precipitaciones y de días con temperaturas entre $\operatorname{los} 16^{\circ} \mathrm{C}$ y $36^{\circ} \mathrm{C}$. Un adecuado ajuste y validación de los modelos predictivos obtenidos de esta investigación, permitieron el desarrollo de verdaderos sistemas de pronóstico para la correcta gestión de la enfermedad, desde el punto de vista técnico, económico y ambiental.

Los distintos modelos epidemiológicos se relacionan estrechamente con las características del patosistema correspondiente. Cada modelo mencionado anteriormente posee características propias que lo definen. En este trabajo, a partir de registros diarios de temperatura máxima y mínima, precipitación y humedad relativa se calcularon variables meteorológicas regresoras en períodos de 15 días previos a cada valor de tasa de incremento diario de severidad, a lo largo de cada curva epidémica. El modelo logístico de respuesta binaria integrado por DPr $>9$ (días con ocurrencia de precipitaciones $>9 \mathrm{~mm}$ ) y DMojro (días de mojado por rocío, sin precipitación y humedad relativa del aire $>82 \%$ ) tuvo una precisión de predicción de $81.5 \%$, permitiendo cuantificar el efecto ambiental en dos tipos de variedades de nogal analizadas. El modelo integrado por DPr $>9$ y DMojt (suma DMojro con los días de mojado por ocurrencia de precipitación (DMojpr)) logró una precisión de predicción de 80,3\%. Ambas variables meteorológicas estarían expresando el requerimiento de agua libre de la bacteria para la infección (DMojt) y de energía en las lluvias (DPr $>9$ ) para favorecer la dispersión bacteriana. Las predicciones de estos modelos pueden sustentar una estrategia para optimizar el número y momento de aplicación de bactericidas cúpricos para un adecuado control de la enfermedad, disminuyendo el impacto ambiental de las pulverizaciones. 


\section{REFERENCIAS}

1. Adaskaveg, J.; Förster, H.; Thompson, D.; Driever, G.; Connell, J.; Buchner, R. Epidemiology and management of walnut blight. Walnut Research Report, Sacramento, CA. p.181-191. 2006

2. Adaskaveg, J.; Förster, H.; Thompson, D.; Driever, .G; Connell, J.; R. Buchner-T. Prichard, J. Grant, and Wade, L. Epidemiology and management of walnut blight. pp185-201. Walnut Research Report Project IV(6):051054. 2007.

3. Adaskaveg, J.; Förster, H.; Nguyen, K.; Thompson, D.; Cary, D.; Wade, L.; Leslie, C.; R. Buchner, R. and Grant, J. Epidemiology and management of Walnut Blight. Walnut Research Reports 1-19. 2016

4. Arquero, O.; Lovera, M.; Rodríguez R. y Trapero A. . Walnut blight (Xanthomonas arboricola pv. Juglandis): factors influencing disease incidence. In: V International Walnut Symposium. 2005, Sorrento, Italia. Anales: ISHS Acta Horticulturae 705, 2005, www.actahort.org/books/705/

5. Belisario, A. Aspetti di eziologia, epidemiologia e difusa delle prinipali avversità patologiche del noce in Italia. Informatore Fitopatologico, Milan, n 7. p.51-57, 2005

6. Bombelli, E.; Wright, E.; Moschini, R.; López, M.; Fabrizio, M.; Barberis, J.; Rivera, M. Modelado computacional de datos epidemiológicos para predecir enfermedades de cultivos con base meteorológica. In: $10^{\circ}$ Simposio sobre la Sociedad de la Información, 41 JAIIO - 2012. La Plata, Argentina. ISSN: 1850-2830- p 322. 2012.

7. Bombelli, E.; Moschini, R.; Wright, E.; López, M.; Fabrizio, M. Modelado para la predicción de enfermedades en cultivos de alto valor comercial. Proyecciones, Buenos Aires, v.11, n.1, p. 47-60, 2013.

8. Costa, L.; Jesus Junior, W.; Ribeiro de Vale, F. Modelos aplicados en fitopatologia. Revista FCA UNCuyo, Mendoza, v.34, n.1, p.81-92, 2002
9. Giraud, M.; Prunet, J.; Chevallier, A.; Ramain, S. ; Thiriaud, V.; I. Santrac, I.; O. Bray, O. Study of Xanthomonas arboricola pv juglandis population dynamics in French Walnut orchards over three years. In: VI Internationa Walnut Symposium. 2010. Melbourne, Australia. ISHS Acta Horticulturae.861, 2010, www.actahort.org/books/861/

10. Lang, M.; Evans, K. Epidemiology and status of Walnut Blight in Australia. Journal of Plant Pathology, Pisa, v.92, (1, Supplement), p.49-55, 2010

11. Lindow, S.; Buchner, R.; Olson, B.; Teviotdale, B.; Koutsoukis, R. Epidemiological approaches to the control of walnut Blight disease. In: Walnut Research Reports 2003.:http://walnutresearch.ucdavis.edu/

12. Lindow, S.; Buchner, R.; Koutsoukis, R. Epidemiological approaches to the control of walnut blight disease. University of California. 200. In: Walnut Research Reports 2007, Junta de Comercializacion de nueces. California. P 221-236. http://walnutresearch.ucdavis.edu/

13. Moschini, R.C.; Canteros, B.; Martínez, M.I.; De Ruyver, R.. Quantification of the environmental effect on citrus canker intensity at increasing distances from a natural windbreak in northeastern Argentina. Australasian Plant Pathol. 43: 653-662. 2014

14. Prichard, T.; Grant, J. and Wade, L. Epidemiology and management of walnut blight. Walnut Research Reports: http://walnutresearch.ucdavis.edu/. 2006.

15. Seta, S.; González, M.; Moyano, M.I. Calidad en poscosecha del nogal (Juglans regia L.). Revista de Investigaciones de la Facultad de Ciencias Agrarias, Rosario. Año IV., n 6., p 51-54., 2004

16. Teviotdale, B.; Michailides, T.; Pscheidt, J. Compendium of nut crop diseases in temperate zones. Minnesota. The American Phytopathological Society Press, 2002.

17. Turini, T. and Teviotdale, B. Epidemiological approaches to the control of Walnut Blight. Experiments of Kearney Agricultural Center 1994. Walnut Research Reports http://walnutresearch.ucdavis.edu/ 1994. 